\title{
The Role of Urinary Neutrophil Gelatinase-Associated Lipocalin in Predicting Acute Kidney Dysfunction in Patients With Liver Cirrhosis
}

\author{
Abdulrahman A. Aljumaha, b, i, Hani Tamimc, Mohamed Saeed ${ }^{a}$, Waleed Tamimi ${ }^{b}, d$, \\ Hanan Alfawaz ${ }^{\mathrm{e}}$, , Salem Al Qurashib, g, Abdulaziz Al Dawood ${ }^{\mathrm{b}, \mathrm{h}}$, \\ Abdulla Al Sayyarib, g
}

\begin{abstract}
Background: Early detection of acute kidney dysfunction (AKD) in cirrhotic patients is crucial. Urinary neutrophil gelatinase-associated lipocalin (uNGAL) has been identified as an early marker of AKD. The aim of the study was to evaluate serial UNGAL as a marker and predictor of AKD in liver cirrhosis patients.
\end{abstract}

Methods: Serial uNGAL and serum creatinine $(\mathrm{sCr})$ levels were measured daily during the first 6 days of admission. Furthermore, $\mathrm{sCr}$ levels and the estimated glomerular filtration rate (eGFR) were measured after $3-6$ weeks. The uNGAL levels in patients with and without abnormal sCr were compared.

Results: Fifty-seven consecutive cirrhotic patients were enrolled in the study. Eight of 14 patients (57\%) who developed abnormal uNGAL level also had abnormal sCr level (odds ratio $(\mathrm{OR})=3.4,95 \%$ CI: $0.99-12.03, \mathrm{P}=0.05)$. After 6 weeks, $41 \%$ of patients exhibited

Manuscript submitted January 25, 2018, accepted February 26, 2018

${ }^{a} H e p a t o l o g y$ Division, Department of Organ Transplant and Hepatobiliary Sciences, King Abdulaziz Medical City, National Guard Health Affairs, Riyadh, Saudi Arabia

${ }^{b}$ King Saud Bin Abdulaziz University for Health Sciences, Ministry of National Guard Health Affairs, Riyadh, Saudi Arabia

${ }^{\mathrm{c}}$ Department of Internal Medicine, American University of Beirut Medical Centre, Beirut, Lebanon

dPathology \& Laboratory Medicine, King Abdulaziz Medical City, National Guard Health Affairs, Riyadh, Saudi Arabia

eDepartment of Food Science and Nutrition, College of Food Science and Agriculture, King Saud University, Riyadh, Saudi Arabia

fPrince Mutaib Chair for Biomarkers of Osteoporosis, King Saud University, Riyadh, Saudi Arabia

gNephrology and Renal Transplantation Division, Department of Medicine, King Abdulaziz Medical City, National Guard Health Affairs, Riyadh, Saudi Arabia

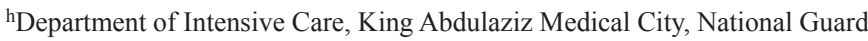
Health Affairs, Riyadh, Saudi Arabia

${ }^{i}$ Corresponding Author: Abdulrahman A. Aljumah, King Abdulaziz Medical City and King Saud Bin Abdulaziz University for Health Sciences, Ministry of National Guard Health Affairs, PO Box 225264, Riyadh 11324, Saudi Arabia. Email:draljumah@hotmail.com

doi: https://doi.org/10.14740/jocmr3366w an abnormal uNGAL level and abnormal $\mathrm{sCr}(\mathrm{OR}=6.7,95 \% \mathrm{CI}$ : $1.55-28.85, \mathrm{P}=0.01$ ). Area under the curve (AUROC) and the best cut-off point for highest NGAL in 6 days were 0.64 and $72.55 \mathrm{ng} / \mathrm{mL}$, respectively.

Conclusions: There is a modest association between highest UNGAL in the first 6 days of admission and $\mathrm{sCr}$ at week 6 in all participants. This may indicate that in cirrhotic patients, uNGAL level during the first 6 days of admission has a potential predictability for the development of high sCr and low eGFR 6 weeks later. The AUROC of 0.64 quantifies the overall ability of UNGAL to discriminate between those individuals who will have a raised $\mathrm{sCr}$ levels and those who will not.

Keywords: Acute kidney dysfunction; Liver cirrhosis; Renal failure; uNGAL; MELD

\section{Introduction}

Acute kidney dysfunction (AKD) including acute kidney injury (AKI) and hepatorenal syndrome (HRS) are commonly occurring in patients with liver cirrhosis. AKD is a life-threatening condition that is associated with poor prognosis. This ranges from mild to severe, which may need to be resolved by renal replacement therapy [1-3].

Two scoring systems are currently used in clinical practice to evaluate the prognosis and severity of patients with liver cirrhosis, namely Child-Turcotte-Pugh (CTP) and the model for end-stage liver disease (MELD) [4-7]. According to CTP, a low score $(5-6)$, moderate score $(7-9)$, and high scores (10 - 15) are associated with good, moderate, and poor prognosis, respectively. For the MELD, a score of $<9,10-39$, and $>$ 40 are associated with good, moderate, and poor prognosis, respectively $[4,5,7]$.

Serum creatinine $(\mathrm{sCr})$ is one of three variables that comprise the MELD score, which is good in predicting the 3 -month mortality and is currently used for prioritization of patients waiting for orthotopic liver transplantation (OLT) [6, 7]. $\mathrm{sCr}$ before OLT has been recently found to be the strongest predictor of survival post-OLT [8]. Moderate-to-severe renal failure at the time of OLT was associated with significantly 
lower short-term and long-term graft and patient survival rates [9].

In a systematic review of 118 studies evaluating predictors of survival in cirrhosis, parameters of liver dysfunction and parameters of AKD were both powerful predictors of death in patients with decompensated cirrhosis [10]. In a previous study, we have shown that using univariate and multivariate analyses, kidney dysfunction is the most significant independent factor for predicting mortality in cirrhotic patients admitted to the intensive care unit [11]. Consequently, early recognition and managing the cause of $\mathrm{AKD}$ in liver cirrhosis are crucial.

Although $\mathrm{sCr}$ is the most widely used test for detection of all types of renal failure, $\mathrm{sCr}$ rises only late after the kidney injury becomes manifest and its level may not reflect the degree of renal damage. Furthermore, $\mathrm{sCr}$ can be affected by other factors such as body weight, race, age, sex, total body volume, drugs, muscle metabolism, and protein intake [12-14]. Additionally, in liver cirrhosis patients, $\mathrm{sCr}$ level is not an accurate test for evaluating kidney function because of the diminished muscle mass associated with this condition. Furthermore, in the presence of high bilirubin level, a low sCr may falsely be reported due to the interference of bilirubin with the $\mathrm{sCr}$ laboratory assay [15].

Estimated glomerular filtration rate (eGFR) is used to assess renal function and is a more sensitive measure of renal impairment than serum creatinine [16]. GFR was found to be associated well with the disruption in kidney function and generally considered the best estimate of renal function $[17$, $18]$.

Many biomarkers have been sought that would help in the early detection of AKD. One promising biomarker is neutrophil gelatinase-associated lipocalin (NGAL), which has been identified using functional genomics and proteomics techniques [19]. NGAL is a $25-\mathrm{kDa}$ ion-transporting protein that is produced in the distal nephron, generally at low concentrations. Its synthesis is up-regulated in response to kidney injury [20]. NGAL is potentially more useful than $\mathrm{sCr}$ as a marker of kidney damage rather than function. NGAL is also a robust marker of kidney disease progression. Serum concentrations of NGAL increase before those of $\mathrm{sCr}$ and it is a powerful tool for monitoring chronic kidney disease (CKD) [21].

NGAL has been investigated and found useful throughout a series of different clinical settings of AKD including cardiac surgery, in critically ill patients, in patients receiving intravenous contrast medium infusion for coronary angiography, and in patients admitted to the emergency department [22-26]. At the time of proposing this study, there was only one published abstract that studied urinary NGAL (uNGAL) in liver cirrhosis, which was later published [27, 28]. More recently, the utility of NGAL as a biomarker in diagnosis of impaired kidney function was investigated in different clinical studies.

In the present study, we aimed to assess the value of $\mathrm{uN}$ GAL in predicting AKD in liver cirrhotic patients. Secondary objectives were to assess the time interval between spikes in uNGAL and AKD and to determine whether a relationship exists between CTP/MELD scores and mean and peak UNGAL concentrations.

\section{Patients and Methods}

\section{Patients}

This is a prospective cohort study in which consecutive adult patients who were admitted to the in-patients wards with the established diagnosis of liver cirrhosis based on liver biopsy, clinical, biochemical and/or radiological ground were included. Patients were excluded from the analysis if they were known to have renal impairment, significant abnormal renal function, CKD stage 3-5, on dialysis and who presented on admission with anuria or have significant proteinuria of more than $300 \mathrm{mg}$ on spot urine. Further, patients were excluded if they were admitted to hospital for shorter than 6 days, admitted to intensive care unit, have a history of kidney transplant, or refused or were unable to provide informed consent.

\section{Ethical statement}

All subjects gave their informed consent for inclusion before they participated in the study. The study was conducted in accordance with the Declaration of Helsinki. The protocol was approved by the institutional review board of our institution.

\section{Methods}

Data were collected including demographic data, etiology of liver disease, complete blood count, liver function parameters, and medication history including diuretics. The septic screening was performed in all patients on admission. uNGAL and sCr were measured daily in patients admitted to our unit during the first 6 days of their admission. Since the uNGAL levels may fluctuate over days for several reasons, we decided to take serial measurements to minimize skewed results. Most of our patients stayed for 2 - 10 days as inpatients. For this reason, we considered including patients who stayed at least 6 days and excluded those who stayed shorter. Urine specimens from patients were evaluated for the presence of uNGAL using the immunoassay analyzer (ARCHITECT) urine NGAL assay (Abbott Laboratories, IL). The upper limit of normal level of uNGAL at our institute's laboratory is $131.7 \mathrm{ng} / \mathrm{mL}$.

The uNGAL levels in those who developed AKD (defined as an acute increase in $\mathrm{sCr}$ level above the normal range and/or a decline in the eGFR below the normal range according to our laboratory reference) were compared to those who did not. In our laboratory, normal $\mathrm{sCr}$ is up to $98 \mu \mathrm{mol} / \mathrm{L}$ in females and up to $110 \mu \mathrm{mol} / \mathrm{L}$ in males. For the calculation of eGFR, we used the four-variable equation from the Modification of Diet in Renal Disease (4-v MDRD) Study: (eGFR (mL/min/1.73 $\left.\mathrm{m}^{2}\right)=186(\mathrm{sCr}$ in $\mu \mathrm{mol} / \mathrm{L} \times 0.011312)-1.154 \times($ age $)-0.203$ $\times(0.742$ if female $))$ [29]. In our laboratory, eGFR of $60 \mathrm{~mL} /$ $\mathrm{min} / 1.73 \mathrm{~m}^{2}$ or higher is in the normal range and eGFR of below 60 indicates kidney disease. 
Table 1. Demographics and Clinical Features of the Study Cohort

\begin{tabular}{ll}
\hline Variables & Mean \pm SD/number (\%) \\
\hline Total sample & $\mathrm{N}=57$ \\
Age & $55.9 \pm 11.0$ \\
Gender & \\
$\quad$ Male & $33(57.9 \%)$ \\
$\quad$ Female & $24(42.1 \%)$ \\
Serum creatinine ( $\mu$ mol/L) (6 days) & $88.5 \pm 37.1$ \\
uNGAL (ng/mL) (6 days) & $69.0 \pm 63.7$ \\
Serum BUN (mmol/L) (2 days) & $7.6 \pm 5.3$ \\
Etiology & $12(21.1 \%)$ \\
$\quad$ Hepatitis B & $26(45.6 \%)$ \\
$\quad$ Hepatitis C & $5(8.8 \%)$ \\
$\quad$ Autoimmune hepatitis & $10(17.5 \%)$ \\
$\quad$ NASH-related & $1(1.8 \%)$ \\
$\quad$ Cryptogenic & $3(5.3 \%)$ \\
$\quad$ Alcohol & \\
CTP & $3(5.3 \%)$ \\
Class A & $24(42.1 \%)$ \\
Class B & $30(52.6 \%)$ \\
Class C & \\
\hline Medigh score (poor prognosis) & $18(31.6 \%)$ \\
\hline
\end{tabular}

SD: standard deviation; NGAL: neutrophil gelatinase-associated lipocalin; BUN: blood urea nitrogen; NASH: non-alcoholic steatohepatitis; CTP: Child-Turcotte-Pugh score; MELD: model for end-stage liver disease score.

Since there is lack of previous studies addressing this hypothesis, it was difficult to conduct a power analysis to gauge cohort size. Cohort size was therefore set based on the estimated number of eligible subjects to be included during the study period $(\mathrm{N}=50)$.

\section{Statistical analyses}

Data collected in this study were entered into a Microsoft Excel sheet specifically structured for the current study. Statistical Analysis Software (SAS version 9.2, SAS Institute, Cary, NC) was used for analyses. Descriptive statistics were carried out by calculating the mean and standard deviation (SD) for the continuous variables, and the number and percent of the categorical variables. Inferential statistics were carried out by using the Student's $t$-test to compare the means of continuous variables between two groups. We used the Fisher exact probability test and the contingency table to calculate the odds ratio (OR) as a measure of risk of development of renal impairment.
Table 2. Correlation Between UNGAL and Serum Creatinine Level at Daily Intervals in All Participants

\begin{tabular}{llll} 
& Serum creatinine level & r & P-value \\
\hline uNGAL & Day 1 & -0.03 & 0.82 \\
uNGAL & Day 2 & 0.17 & 0.22 \\
uNGAL & Day 3 & 0.03 & 0.02 \\
uNGAL & Day 4 & 0.49 & 0.00 \\
uNGAL & Day 5 & 0.48 & 0.001 \\
uNGAL & Day 6 & 0.13 & 0.49 \\
\hline
\end{tabular}

UNGAL: urinary neutrophil gelatinase-associated lipocalin; r: correlation coefficient.

Pearson correlation coefficient was used to assess the association between two continuous variables. We used area under the curve (AUROC) analysis as a measure of the predictive capability of uNGAL for the development of abnormal sCr. The best cut-off point was identified using the Youdens index. Moreover, sensitivity and specificity were also calculated. Statistical significance was defined by $\mathrm{P}$-value of $\leq 0.05$.

\section{Results}

Fifty-seven consecutive cirrhotic patients met the inclusion criteria and were enrolled. The mean age was $55.9 \pm 11.0$ years, and 58\% was male. The most frequent cause of liver cirrhosis was hepatitis $\mathrm{C}$ virus (45.6\%). Most patients had advanced liver disease as indicated by high CTP (52.6\%) and MELD (31.6\%). The demographics and clinical and laboratory data are shown in Table 1.

The reasons for admission were: 19 patients with hepatic encephalopathy, 10 with spontaneous bacterial peritonitis, nine with hepatocellular carcinoma admitted for tumor control, 10 for liver transplantation workup, six due to severe ascites with or without pleural effusion for management, and three for variceal bleeding control. Fourteen out of 57 patients $(25 \%)$ were found to have an abnormally raised UNGAL level at least once during the first 6 days of admission. Twenty out of 57 patients $(35 \%)$ developed abnormal $\mathrm{sCr}$ at least one time during the first 6 days of admission. Eight of the 14 patients (57\%) who developed abnormal uNGAL level concomitantly presented with abnormal sCr level (OR $=3.4,95 \% \mathrm{CI}$ : $0.99-12.03, \mathrm{P}=$ $0.053)$. The correlation between $\mathrm{UNGAL}$ and $\mathrm{sCr}$ levels in all participants is shown in Table 2 and Figure 1.

Out of the 57 patients, positive bacterial cultures were reported from five urine, two blood and seven ascetic fluid cultures. Of those 14 patients, only two have blood and urine infection simultaneously. However, only two of the patients with urinary tract infection and two of the patients with bacteremia had an abnormal uNGAL level. On testing the validity of UNGAL as a predictor for the development of abnormal $\mathrm{sCr}$, it was found that the AUROC and the best cut-off point for highest NGAL in 6 days (considering abnormal $\mathrm{sCr}$ as a positive state variable) were 0.64 and 72.55 , respectively (Fig. 2). uNGAL level during the first 6 days as a test to predict development of abnormal $\mathrm{sCr}$ has a sensitivity of $40 \%(95 \%$ 

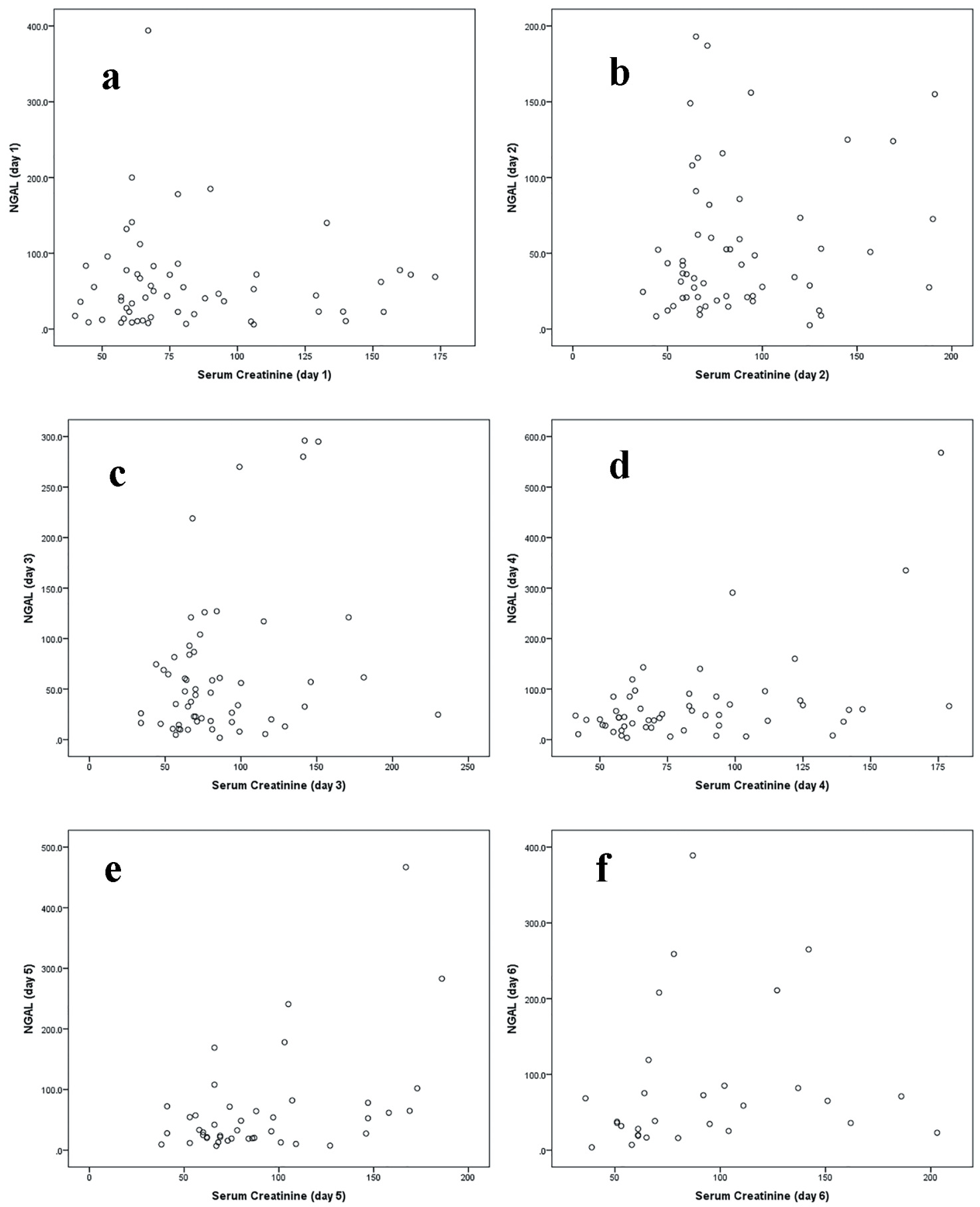

Figure 1. Correlation between uNGAL and serum creatinine level at daily intervals in all participants (a, b, c, d, e, f).

CI: $19.2-63.9 \%)$, a specificity of $84 \%$ (95\% CI: 67.9-93.8\%), a positive predictive value (PPV) of $57 \%$ (95\% CI: 28.9-82.2\%), and a negative predictive value (NPV) of $72 \%$ (95\% CI: $56.3-$ $84.7 \%$ ). After 6 weeks of follow-up, 22 out of the available 54 patients had developed abnormal sCr level. Nine of these $(40.9 \%)$ had a raised uNGAL level at the first 6 days of admission $(\mathrm{OR}=6.7,95 \% \mathrm{CI}: 1.55-28.85, \mathrm{P}=0.01)$. Likewise, out of the 54 patients available for follow-up and subsequent anal- ysis, 22 patients developed a decline in their eGFR at 6 weeks. Nine of them $(40.9 \%)$ had high uNGAL during the first few days $(\mathrm{OR}=6.7,95 \% \mathrm{CI}: 1.55-28.85, \mathrm{P}=0.01)$. In addition, we also found a significant association between highest NGAL in 6 days and $\mathrm{sCr}$ at week 6 in all participants. The mean $\pm \mathrm{SD}$ of the highest uNGAL in 6 days was $73.6 \pm 71.1$ and $145.1 \pm$ $145.0(\mathrm{P}=0.04)$ for normal and abnormal $\mathrm{sCr}$, respectively.

At 6 weeks follow-up, the sensitivity and NPV had not 


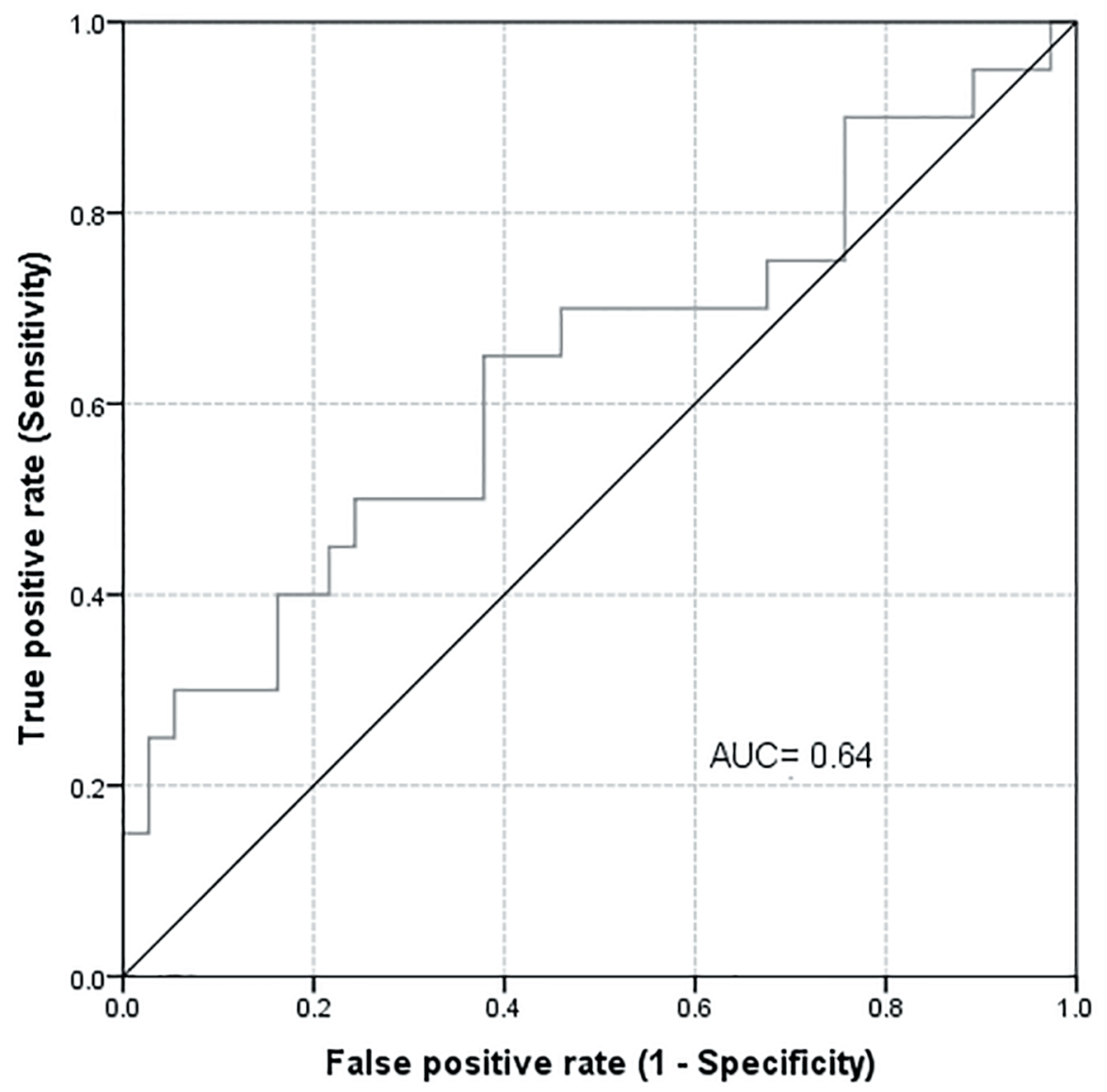

Figure 2. Area under the curve for highest UNGAL in 6 days considering abnormal creatinine as a positive state variable.

changed (40.9\%, 95\% CI: 20.8-63.6\% and 69.1\%, 95\% CI: $52.9-82.4 \%$, respectively). However there was a substantial improvement in the specificity and PPV $(90.7 \%, 95 \%$ CI: $75.1-$ $97.9 \%$ and $75.0 \%, 95 \% \mathrm{CI}: 42.8-94.2 \%$ ). These results indicate an important association between the development of high uNGAL level and the development of AKD. This association was more noticeable at 6 weeks of follow-up. If we consider the abnormal estimated glomerular filtration rate (eGFR) as a positive state variable, the AUROC will improve marginally to 0.661 with the best cut off point at 63.60 (Fig. 3).

We further performed a calculation for the correlation between the high uNGAL level at the first 6 days of admission and the low eGFR at 6 weeks of follow-up. We found a negative correlation which means for each increase in UNGAL at first 6 days of admission, there is a decline in eGFR at 6 weeks of follow-up, where it was significant for the day 2 (correlation $=-0.34, \mathrm{P}$-value $=0.01)$, day 3 (correlation $=-0.30, \mathrm{P}$-value $=$ $0.03)$, day 4 (correlation $=-0.30, \mathrm{P}$-value $=0.04)$, day 5 (correlation $=-0.34$, P-value $=0.03)$, and day 6 (correlation $=-0.37$, P-value =0.06) (Fig. 4).

A multivariate analysis for the possibility of highest $\mathrm{uN}$ GAL measured during the first 6 days of admission as a predictor for the development of high $\mathrm{sCr}$ and low eGFR at 6 weeks later was performed after controlling for the different confounders (age, gender, CTP, MELD, hemoglobin and Albu- $\min )$. There was an association between highest uNGAL during the first 6 days and the development of high sCr at 6 weeks later $(\mathrm{OR}=1.06,95 \% \mathrm{CI}: 0.98-1.15, \mathrm{P}=0.14$. On the other hand, an association was also found between the highest uNGAL during the first 6 days and the development of low eGFR at 6 weeks later $(\mathrm{OR}=1.06,95 \% \mathrm{CI}: 0.98-1.14, \mathrm{P}=0.13)$.

There was no correlation between NGAL and severity of liver disease as evaluated by either CTP or MELD scores. Although MELD had a better correlation than CTP, the difference was not statistically significant $(P=0.17$ versus $P=0.43$, respectively).

\section{Discussion}

Patients with liver cirrhosis are vulnerable to the development of AKD. Several mechanisms were postulated: hypovolemia and intravascular depletion induced by diuretics, paracentesis, gastrointestinal bleeding and infection may lead to pre-renal azotemia and acute tubular necrosis (ATN) [30, 31]. Hepatorenal syndrome is another potential cause for $\mathrm{AKD}$ in cirrhotic patients.

Physicians managing patients with liver and kidney diseases are in desperate need for a test that can predict the development of AKD before its occurrence. This will lead to an 


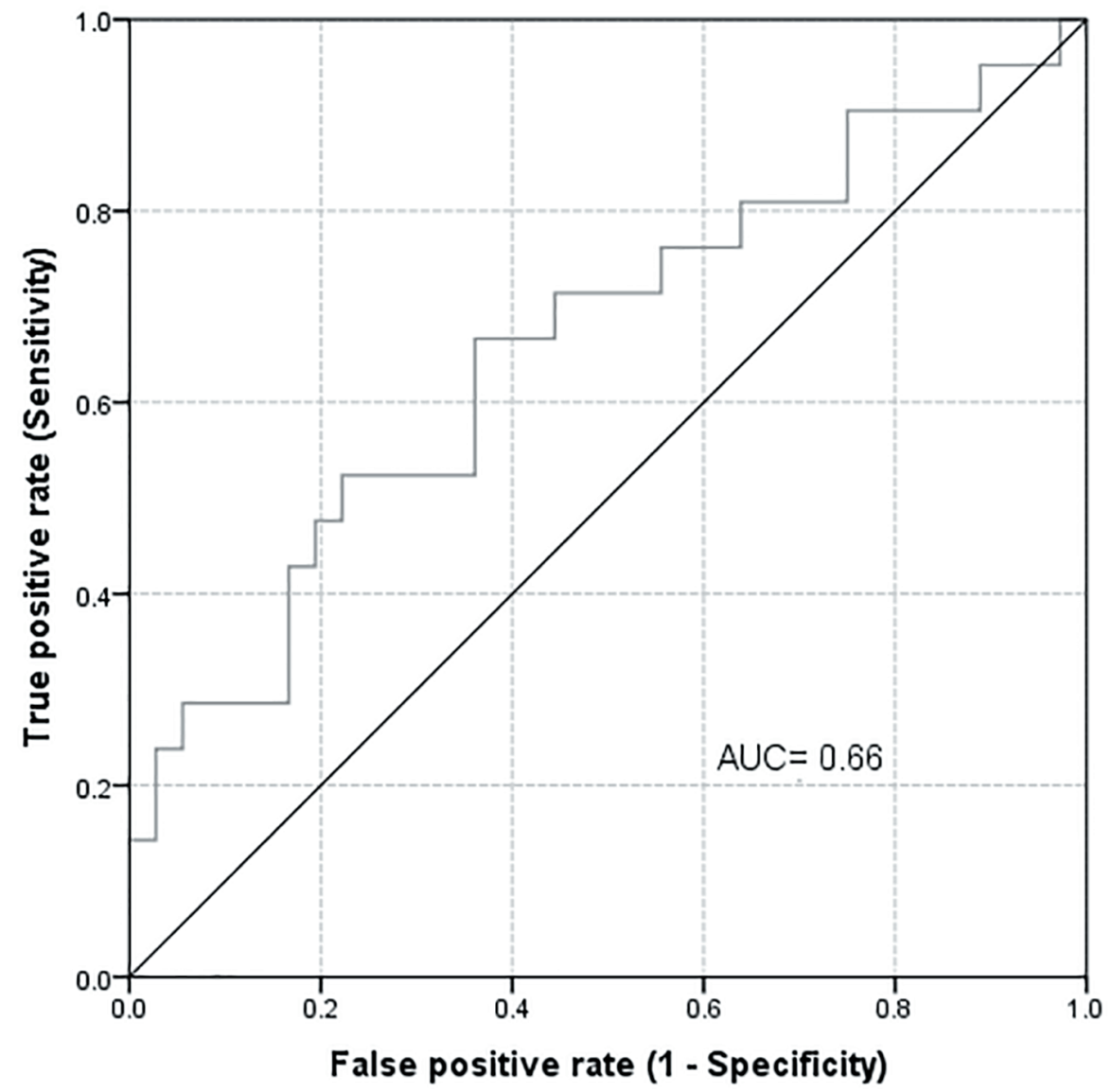

Figure 3. Area under the curve for highest UNGAL in 6 days considering abnormal eGFR as a positive state variable.

immediate intervention that treats and prevent the occurrence of AKD.

In this study, we found that eight of the 14 patients (57\%) who developed abnormal uNGAL level also had a concomitant abnormal sCr. Although this suggests an association between high uNGAL and abnormal sCr during the first 6 days of admission, it did not reach statistical significance $(\mathrm{OR}=3.4$, $95 \%$ CI: $0.99-12.03, \mathrm{P}=0.05)$. Two recent studies examined uNGAL levels in liver cirrhosis patients with and without ascites and AKD or HRS and evaluated its role as a predictor of mortality $[25,28]$. uNGAL level was significantly higher in those with impaired kidney function, urinary tract infections, CKD and HRS. In a study by Fagundes et al [32], patients with impaired kidney function had higher uNGAL levels compared to patients with and without ascites. Patients with ATN had markedly higher uNGAL levels compared to those of patients with prerenal azotemia due to volume depletion, CKD and HRS ( $\mathrm{P}<0.001)$. In Verna et al's study [28], uNGAL levels discriminated type of AKD. UNGAL levels were highest in patients with AKI, significantly different from patients with normal kidney function, stable CKD, prerenal azotemia and HRS. In HRS, uNGAL was intermediate between, and significantly different from, patients either with AKD $(\mathrm{P}<0.001)$ or prerenal azotemia $(P=0.004)$. In patients with prerenal azotemia, uNGAL levels were low and equivalent to levels in patients with normal kidney function and stable CKD; no patient with prerenal azotemia had an uNGAL level > $100 \mathrm{ng} / \mathrm{mL}$ [28].

In a recent study by El-Bassat et al [33] on liver cirrhosis patients, it revealed that patients with impaired kidney function had significantly higher uNGAL levels compared with those with normal kidney function and healthy control $(\mathrm{P}=$ 0.001). Patients with HRS had a significantly higher level of uNGAL compared with those with prerenal azotemia $(\mathrm{P}=$ $0.01)$, CKD ( $\mathrm{P} \leq 0.05)$, or cirrhotic patients with normal kidney function $(\mathrm{P}=0.01)$. The highest values of UNGAL were detected in patients with AKD $(\mathrm{P}=0.001)$ [33]. Our results on the relation between the high level of uNGAL and $\mathrm{sCr}$ were similar to those of Barreto et al. They found patients with AKD had significantly higher uNGAL levels compared to those of patients without AKD $(203 \pm 390$ vs. $79 \pm 126 \mu \mathrm{g} / \mathrm{g}$ of $\mathrm{sCr}, \mathrm{P}$ $<0.001$ ), nevertheless there was an overlap between the two groups of patients [34].

In another study on cirrhotics, patients with renal impairment had higher uNGAL levels $(357.78 \pm 228.51 \mu \mathrm{g} / \mathrm{g} \mathrm{sCr})$ compared to those of patients without renal impairment, either with or without ascites $(96.84 \pm 35.58,113.76 \pm 47.98 \mu \mathrm{g} / \mathrm{g}$ sCr, respectively) [35].

In a recent study, we confirmed that uNGAL concentrations measured during the first day after kidney transplant may be useful in predicting the development of delayed graft func- 

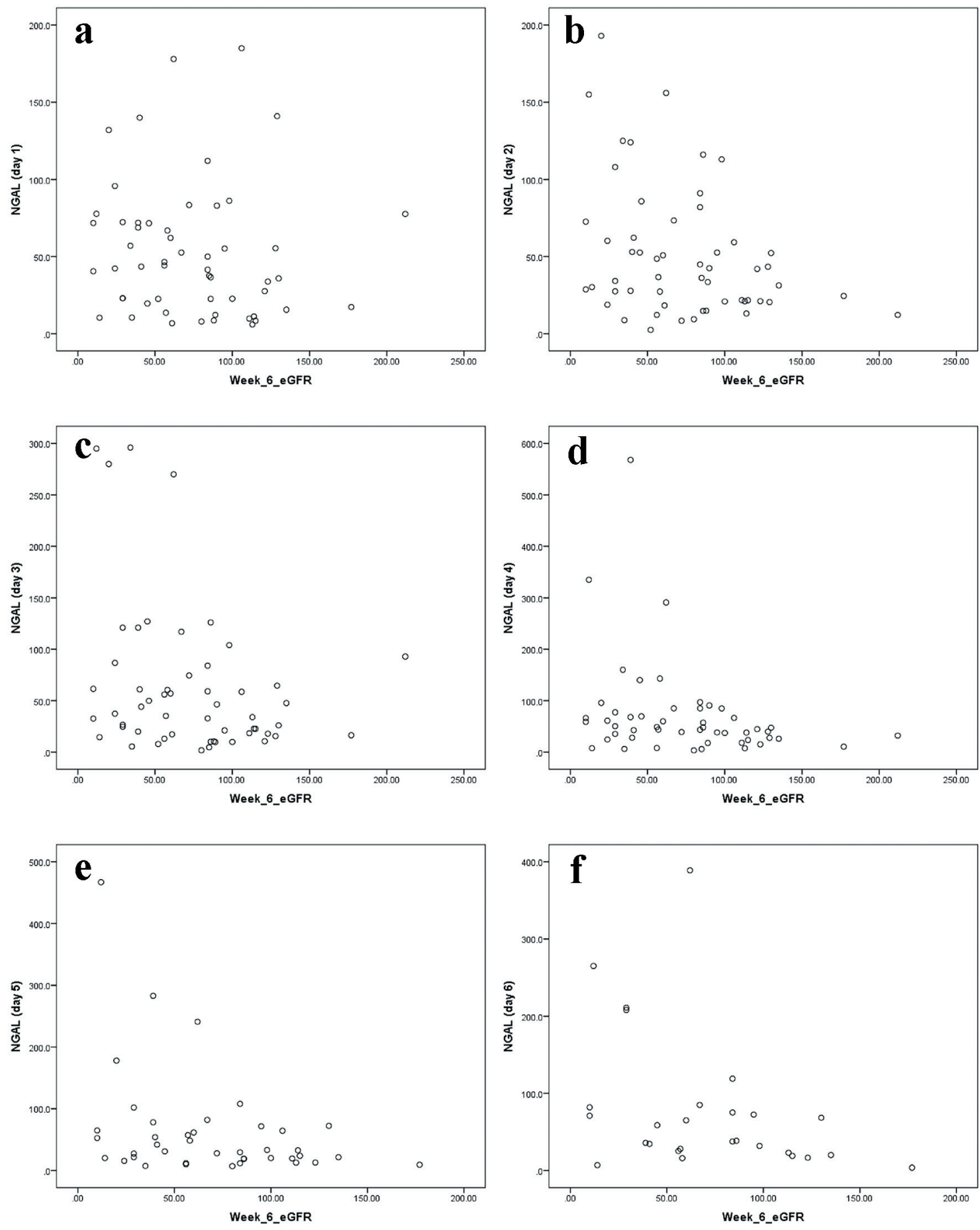

Figure 4. Correlation between high uNGAL level at the first 6 days of admission and low eGFR at 6 weeks of follow-up (a, b, c, d, e, f).

tion. In addition, uNGAL measured in the donor kidney perfusate also may help predict the development of delayed graft function and decreased short-term graft function [36]. In our current study, one patient died and two patients were lost to follow-up after 6 weeks of follow-up. Out of the remaining 54 patients, 22 have had a significant rise in their sCr. Nine of these $(40.9 \%)$ had a concomitant abnormal uNGAL level $(\mathrm{OR}=6.7,95 \% \mathrm{CI}: 1.55-28.85, \mathrm{P}=0.010)$. These finding may indicate the possible utility of UNGAL measurement during first few days of admission in predicting the development of AKD several weeks later. Measuring serials of UNGAL on consecutive days has allowed us to detect the abnormal levels 
of UNGAL that may fluctuate from day to day due to several unknown confounding factors. We further followed $\mathrm{sCr}$ level after 6 weeks of admission. None of the previously published studies has followed the $\mathrm{sCr}$ for a longer time after admission. We also found a significant association between highest NGAL in 6 days and sCr at week 6 in all participants. We performed testing of the validity of UNGAL as a predictor for the development of abnormal sCr. We found that when we considered abnormal $\mathrm{sCr}$ as a positive state variable, that AUROC of 0.64 quantifies the overall ability of UNGAL to discriminate between those individuals with abnormal and those with normal $\mathrm{sCr}$ and the best cut-off point for highest NGAL in 6 days was 72.55. However, the AUROC of 0.64 was not very high and may indicate only a modest predictability. After 6 weeks of follow-up, the sensitivity and NPV did not change; however, there were significant improvements in the specificity and PPV. Verna et al performed AUROC analysis to test each biomarker's ability to identify patients with AKD. UNGAL and $\mathrm{sCr}$ similarly discriminated AKD (AUC 0.86 vs. 0.89 , respectively, $\mathrm{P}=0.6)$. uNGAL discriminated AKD better than fractional excretion of sodium (AUC 0.71, $\mathrm{P}=0.1$ ), though this difference was not significant. At a cut-off of $110 \mathrm{ng} / \mathrm{mL}$, uNGAL was $88 \%$ sensitive and $85 \%$ specific for the diagnosis of AKD [28]. In the study by Barreto et al [34], the AUROC of uNGAL for detecting AKD was 0.724 (0.637 - 0.811). The value of uNGAL, which better, discriminated between presence and absence of AKD was $51 \mu \mathrm{g} / \mathrm{g}$ of $\mathrm{sCr}$ (sensitivity $66 \%$, specificity 70\%). El-Bassat et al [33] found similar results. They found that a cut-off value of $110 \mathrm{ng} / \mathrm{mL}$ uNGAL had sensitivity, specificity, PPV, NPV, and accuracy of $90.2 \%, 67.9 \%$, $79.0 \%, 91 \%$, and $88.75 \%$, respectively, to diagnose HRS [33]. In Ahmed et al's study, the cut-off value of uNGAL that differentiates between patients with AKD and those with other causes of renal impairment was $286.3 \mu \mathrm{g} / \mathrm{g} \mathrm{sCr}$ (AUROC is 0.909 ) (sensitivity $95.5 \%$ and specificity $76.1 \%$ ) with PPV of 65.6 and NPV of 99.2. uNGAL demonstrated an excellent discriminating power compared to $\mathrm{SCr}$ (AUROC 0.909 for NGAL and 0.622 for $\mathrm{Cr}$ ) for discriminating ATN from HRS [35]. In our current study, when we consider the abnormal eGFR as a positive state variable, the AUROC improved marginally to 0.661 with the best cut-off point at 63.60 ; however, this has only a modest predictability effect. In a small cross-sectional study, Gerbes et al [37] evaluated the relationship between measured glomerular filtration rate (mGFR) with plasma and UNGAL in patients with decompensated cirrhosis and variable kidney function. They found discordance between mGFR and eGFR in patients with cirrhosis that the latter was significantly higher. They also showed that a cut-off of plasma NGAL > $100 \mathrm{ng} / \mathrm{mL}$ was superior compared with $\mathrm{sCr}$ to predict patients with $\mathrm{mGFR}<50 \mathrm{~mL} / \mathrm{min}$. In our study, we found no correlation between NGAL and severity of liver disease as evaluated by either CTP or MELD scores. Although MELD had a better correlation than CTP, the difference was not statistically significant $(\mathrm{P}=0.17$ versus 0.43 , respectively). Our study has several limitations: first, it has relatively small sample size; second, the study was conducted in a single center. Exclusion of those with preexisting renal dysfunction and patients who required ICU admission has further reduced our sample size. Nonetheless, our study demonstrated that uNGAL is a poten- tially useful predictor of AKD. One of the great advantages of our study was that we had obtained serial uNGAL measurements, which were not done by previous investigators. We believe that this could be useful in the early prediction of AKD. Larger multicenter studies are needed for more robust data to confirm the utility of UNGAL. With the introduction of formulas that calculate the eGFR, the screening for any kidney damage has become easily available. In this study, we utilized (4-v MDRD) formula, which has been recommended by several international scientific societies. It was postulated that this formula is the best available equation for calculating eGFR and it enhances the validity of serum creatinine in detecting renal dysfunction [38].

\section{Conclusions}

In this study, we found that in liver cirrhosis patients, there was a modest association between the development of high uNGAL level at the early few days of admission and the development of high $\mathrm{sCr}$ and low eGFR. This association was more noticeable at 6 weeks of follow-up. The AUROC of 0.635 quantifies the overall ability of UNGAL to discriminate between those individuals with abnormal and those with normal sCr. However, AUROC of 0.64 has only a modest effect and may not indicate a highly significant prediction rate. There was no correlation between NGAL and severity of liver disease as calculated based on either CTP or MELD scores. This study revealed that patients with impaired kidney function had higher uNGAL levels compared with those without impairment of kidney function. From the above results, we conclude that in cirrhotic patients, uNGAL level during the first 6 days of admission may have a modest prediction for the development of kidney dysfunction as manifested by high $\mathrm{sCr}$ and low eGFR 6 weeks later.

\section{Conflict of Interest}

The authors declare no conflict of interest.

\section{Grant Support}

This project was funded by King Abdullah International Medical Research Center, Ministry of National Guard Health Affairs, Riyadh, Saudi Arabia.

\section{References}

1. Webb S, Dobb G. ARF, ATN or AKI? It's now acute kidney injury. Anaesth Intensive Care. 2007;35(6):843-844.

2. Mehta RL, Kellum JA, Shah SV, Molitoris BA, Ronco C, Warnock DG, Levin A, et al. Acute Kidney Injury Network: report of an initiative to improve outcomes in acute kidney injury. Crit Care. 2007;11(2):R31.

3. Rahman M, Shad F, Smith MC. Acute kidney injury: a 
guide to diagnosis and management. Am Fam Physician. 2012;86(7):631-639.

4. Cholongitas E, Papatheodoridis GV, Vangeli M, Terreni N, Patch D, Burroughs AK. Systematic review: The model for end-stage liver disease-should it replace ChildPugh's classification for assessing prognosis in cirrhosis? Aliment Pharmacol Ther. 2005;22(11-12):1079-1089.

5. Kamath PS, Wiesner RH, Malinchoc M, Kremers W, Therneau TM, Kosberg CL, D'Amico G, et al. A model to predict survival in patients with end-stage liver disease. Hepatology. 2001;33(2):464-470.

6. Garcia-Tsao G, Parikh CR, Viola A. Acute kidney injury in cirrhosis. Hepatology. 2008;48(6):2064-2077.

7. Wiesner R, Edwards E, Freeman R, Harper A, Kim R, Kamath P, Kremers W, et al. Model for end-stage liver disease (MELD) and allocation of donor livers. Gastroenterology. 2003;124(1):91-96.

8. Weismuller TJ, Prokein J, Becker T, Barg-Hock H, Klempnauer J, Manns MP, Strassburg CP. Prediction of survival after liver transplantation by pre-transplant parameters. Scand J Gastroenterol. 2008;43(6):736-746.

9. Nair S, Verma S, Thuluvath PJ. Pretransplant renal function predicts survival in patients undergoing orthotopic liver transplantation. Hepatology. 2002;35(5):1179-1185.

10. D’Amico G, Garcia-Tsao G, Pagliaro L. Natural history and prognostic indicators of survival in cirrhosis: a systematic review of 118 studies. J Hepatol. 2006;44(1):217231.

11. Arabi Y, Ahmed QA, Haddad S, Aljumah A, Al-Shimemeri A. Outcome predictors of cirrhosis patients admitted to the intensive care unit. Eur J Gastroenterol Hepatol. 2004;16(3):333-339.

12. Star RA. Treatment of acute renal failure. Kidney Int. 1998;54(6):1817-1831.

13. Tomlanovich S, Golbetz H, Perlroth M, Stinson E, Myers BD. Limitations of creatinine in quantifying the severity of cyclosporine-induced chronic nephropathy. Am J Kidney Dis. 1986;8(5):332-337.

14. Baboolal K, Jones GA, Janezic A, Griffiths DR, Jurewicz WA. Molecular and structural consequences of early renal allograft injury. Kidney Int. 2002;61(2):686-696.

15. Dimeski G, McWhinney B, Jones B, Mason R, Carter A. Extent of bilirubin interference with Beckman creatinine methods. Ann Clin Biochem. 2008;45(Pt 1):91-92.

16. Thomas C, Thomas L. Renal failure - measuring the glomerular filtration rate. Dtsch Arztebl Int. 2009;106(5152):849-854.

17. Stevens LA, Levey AS. Measured GFR as a confirmatory test for estimated GFR. J Am Soc Nephrol. 2009;20(11):2305-2313.

18. Sandilands EA, Dhaun N, Dear JW, Webb DJ. Measurement of renal function in patients with chronic kidney disease. Br J Clin Pharmacol. 2013;76(4):504-515.

19. Wu I, Parikh CR. Screening for kidney diseases: older measures versus novel biomarkers. Clin J Am Soc Nephrol. 2008;3(6):1895-1901.

20. Ronco C. N-GAL: diagnosing AKI as soon as possible. Crit Care. 2007;11(6):173.

21. Bachorzewska-Gajewska H, Malyszko J, Sitniewska E,
Malyszko JS, Pawlak K, Mysliwiec M, Lawnicki S, et al. Could neutrophil-gelatinase-associated lipocalin and cystatin $\mathrm{C}$ predict the development of contrast-induced nephropathy after percutaneous coronary interventions in patients with stable angina and normal serum creatinine values? Kidney Blood Press Res. 2007;30(6):408-415.

22. Dent CL, Ma Q, Dastrala S, Bennett M, Mitsnefes MM, Barasch J, Devarajan P. Plasma neutrophil gelatinase-associated lipocalin predicts acute kidney injury, morbidity and mortality after pediatric cardiac surgery: a prospective uncontrolled cohort study. Crit Care. 2007;11(6):R127.

23. Nickolas TL, O’Rourke MJ, Yang J, Sise ME, Canetta PA, Barasch N, Buchen C, et al. Sensitivity and specificity of a single emergency department measurement of urinary neutrophil gelatinase-associated lipocalin for diagnosing acute kidney injury. Ann Intern Med. 2008;148(11):810819.

24. Zappitelli M, Washburn KK, Arikan AA, Loftis L, Ma Q, Devarajan P, Parikh CR, et al. Urine neutrophil gelatinase-associated lipocalin is an early marker of acute kidney injury in critically ill children: a prospective cohort study. Crit Care. 2007;11(4):R84.

25. Ostermann M, Chang RW. Acute kidney injury in the intensive care unit according to RIFLE. Crit Care Med. 2007;35(8):1837-1843; quiz 1852.

26. Lacquaniti A, Buemi F, Lupica R, Giardina C, Mure $\mathrm{G}$, Arena A, Visalli C, et al. Can neutrophil gelatinaseassociated lipocalin help depict early contrast materialinduced nephropathy? Radiology. 2013;267(1):86-93.

27. Verna E, Farrand E, Pichardo E, et al. Urinary neutrophil gelatinase associated lipocalin (uNGAL) distinguishes type of kidney failure and predicts mortality in patients with cirrhosis. [ABSTRACT]. Hepatology. 2009;50(Suppl):311A.

28. Verna EC, Brown RS, Farrand E, Pichardo EM, Forster CS, Sola-Del Valle DA, Adkins SH, et al. Urinary neutrophil gelatinase-associated lipocalin predicts mortality and identifies acute kidney injury in cirrhosis. Dig Dis Sci. 2012;57(9):2362-2370.

29. Levey AS, Coresh J, Greene T, Stevens LA, Zhang YL, Hendriksen S, Kusek JW, et al. Using standardized serum creatinine values in the modification of diet in renal disease study equation for estimating glomerular filtration rate. Ann Intern Med. 2006;145(4):247-254.

30. Betrosian AP, Agarwal B, Douzinas EE. Acute renal dysfunction in liver diseases. World J Gastroenterol. 2007;13(42):5552-5559.

31. Newell GC. Cirrhotic glomerulonephritis: incidence, morphology, clinical features, and pathogenesis. Am J Kidney Dis. 1987;9(3):183-190.

32. Fagundes C, Pepin MN, Guevara M, Barreto R, Casals G, Sola E, Pereira G, et al. Urinary neutrophil gelatinaseassociated lipocalin as biomarker in the differential diagnosis of impairment of kidney function in cirrhosis. J Hepatol. 2012;57(2):267-273.

33. El-Bassat H, Ziada DH, Taha A, Alm-Eldin R. Urinary neutrophil gelatinase-associated lipocalin as a biomarker for the diagnosis of hepatorenal syndrome in cirrhotic patients. Tanta Med J. 2013;41:346-352. 
34. Barreto R, Elia C, Sola E, Moreira R, Ariza X, Rodriguez E, Graupera I, et al. Urinary neutrophil gelatinaseassociated lipocalin predicts kidney outcome and death in patients with cirrhosis and bacterial infections. J Hepatol. 2014;61(1):35-42.

35. Ahmed QA, El Sayed FS, Emad H, Mohamed E, Ahmed B, Heba P. Urinary biomarkers of acute kidney injury in patients with liver cirrhosis. Med Arch. 2014;68(2):132136.

36. Qurashi S, Ghamdi G, Jaradat M, Tamim H, Aljumah A, Tamimi W, Al Dawood A, et al. Urinary neutrophil gelati- nase-associated lipocalin and the occurrence of delayed graft function after kidney transplant. Exp Clin Transplant. 2014;12(5):396-400.

37. Gerbes AL, Benesic A, Vogeser M, Krag A, Bendtsen F, Moller S. Serum neutrophil gelatinase-associated lipocalin - a sensitive novel marker of renal impairment in liver cirrhosis? Digestion. 2011;84(1):82-83.

38. Lamb EJ, Tomson CR, Roderick PJ, Clinical Sciences Reviews Committee of the Association for Clinical B. Estimating kidney function in adults using formulae. Ann Clin Biochem. 2005;42(Pt 5):321-345. 